\section{Physiological and psychological correlates of personality impression formation}

JOHN T. PARTINGTON and FRANK D. COLMAN, University of Western Ontario, Ont., Canada

This preliminary study of strategies involved in personality-impression formation utilized complex stimuli and novel dependent variables, inchuding pupillary activity, syntactic features of verbalizations, and multidimensional trait ratings. Two recurring strategies were found that appear to reflect different degrees of conceptual complexity. Interpretations based on observed contiguous associations between pupillary activity and stimulus information were supported by correlational evidence from the verbalizations and trait ratings.

Forming an impression of someone is a cognitive task that requires us to process various kinds of information. Asch (1946) initiated investigation of this phenomenon more than 2 decades ago However, the majority of subsequent work has involved simply testing the "goodness of fit" of arithmetic models against obtained inferential judgments. As a result, a host of competing formulations have emerged (e.g., averaging vs adding), with the seemingly inevitable paradox of high prediction with little understanding. This state of affairs is not surprising, considering that most studies have employed "rigorous" designs that represented hypothetical stimulus people by means of unidimensional sets of trait adjectives and obtained simple evaluative ratings as dependent variables.

The purpose of the present study was to increase understanding about impression formation by focusing on individual processing strategies (cf. Schroder, Driver, \& Streufert, 1967). Complex stimuli were used, and a wide array of response measures were obtained, including a psychophysiological response, pupillary dilation, and syntactic features of verbal responses, together with a multidimensional set of trait-rating scales. Pupillary dilation, perhaps the most novel measure in this battery, has never been used as a dependent variable to study personality-impression formation. It was chosen because of its demonstrated sensitivity for reflecting information processing. For example, pupillary dilation has been related to the difficulty of solving multiplication tasks (Hess \& Polt, 1964), generating "mental images" to words differing in concreteness (Paivio \& Simpson, 1966), and to the difficulty of storing, in memory, task information of various kinds (Kahneman \& Beatty, 1966).

The Ss were 27 introductory psychology students who had no visual defects. This sample was subsequently reduced to 20,10 male and 10 female. Four Ss were eliminated because their pupils were out of focus on the film, two because of excessive blinking, and one male $\mathbf{S}$ was randomly discarded to equate the number of males and females in the final sample.

A quantified, but realistic, set of stimulus characteristics was needed in order to study how Ss would process information about another. Accordingly, the following six self-referent statements were used to represent a stimulus person. They were selected from the Personality Research Form (Jackson, 1967) because their operating characteristics reflected high denotative relevance and minimum connotations of desirability (mean absolute deviation of item values from neutral: denotation $\bar{X}=2.2$; desirability $\bar{X}=0.9$, $t=3.8, p<.01)$ :

I try to control others rather than letting them control me: I pay little attention to the interests of people I know: If I have to pack a suitcase I usually organize it very well: I usaally let others take the lead and go along with their ideas: I need the feeling of belonging that comes from having many friends: I have a lot of trouble keeping an accurate record of my expenses.

The items denote opposite poles of three personality dimensions: dominance, affiliation, and orderliness. These dimensions were considered most appropriate since they represent the major orthogonal components in people's implicit personality theories (Lay \& Jackson, 1968). Conflicting information was used so that the stimuli would be complex enough to insure the emergence of individual response styles.

The description of the experimental stimulus person was presented aurally through earphones that were connected to a two-channel tape recorder that also controlled the different time intervals used in the study. The apparatus for recording pupillary dilations consisted of a $16-\mathrm{mm}$ camera mounted on a box-like compartment (cf. Paivio \& Simpson, 1966). The S was seated at the open end of this box and fixated a point on a screen at the opposite end. Photographs of his right eye were taken at a filming speed of 2 frames/sec. His verbal remarks were recorded by means of a second tape recorder. The written testing materials comprised a set of bipolar 7-point scales, some of which denoted personality characteristics directly elevant to the experimental stimulus person (e.g., strong-weak, friendly-unfriendly, orderly-disorderly), while others suggested additional connotations (e.g., secure-insecure, acceptable-unacceptable).

The Ss were tested individually. Each S was told that the experiment would deal with how people form impressions of others, and that the camera would make it possible to study indirectly his thinking processes during the task. He was asked to put on the earphones and listen to the instructions. These informed him that he would hear six self-referent statements made by a person. He was told that this information was to be used to form an impression of that person, which he would subsequently have to verbalize. After the camera was started, an example trial was provided to familiarize the $S$ with the situation. This involved a $30-\mathrm{sec}$ rest interval followed by a $60-\mathrm{sec}$ stimulus presentation phase, a $90-\mathrm{sec}$ response interval, and another $30-\mathrm{sec}$ rest period. The $S$ was alerted for the experimental trial by a "ready" signal followed by a 10-sec pause. The six statements representing the experimental stimulus person were then presented at $10-\mathrm{sec}$ intervals. Each statement took approximately $5 \mathrm{sec}$ to present and was followed by a 5-sec pause. The same presentation order was used for all Ss so that their pupillary reactions to each statement might be compared. This 60-sec stimulus-presentation phase was followed by a $90-\sec$ period during which the $S$ verbalized his impression of the stimulus person. This verbal response was tape-recorded for subsequent content analysis. Finally, the tape recording of the stimulus statements was replayed and the $S$ was asked to judge the experimental stimulus person on the rating scales provided.

\section{DISCUSSION}

The analysis focused on how these Ss processed information when forming impressions of the stimuhus person. Data comprised a physiological correlate of information processing, i.e., $120 \mathrm{Fim}$ exposures of each S's pupil during the task, and verbalized impression and trait ratings of the stimulus person. The general approach was to seek out recurring strategies of information processing as reflected in pupillary activity, and to attempt to interpret these in relation to contiguously associated stimuli and also in relation to the other response measures.

The first step in the search for common 


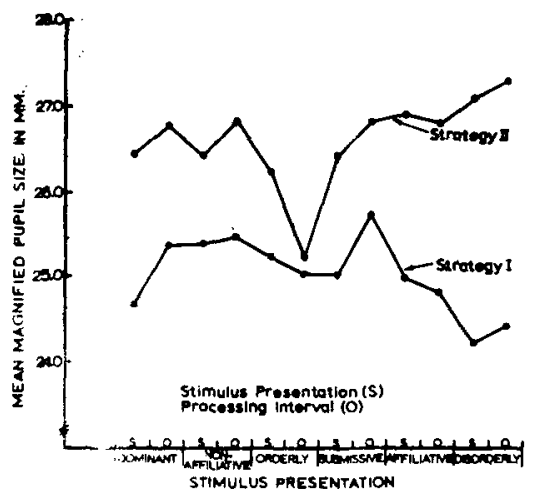

Fig. 1. Strategies in personalityimpression formation reflected by mean pupiliary response curves as a function of stimulus information.

processing strategies involved forming a profile of each S's pupillary reactions. The film of aach S's pupil was magnitide and pupil size was measured in millimeters. These measurements were then blocked into 12 segments, 6 reflecting information processing during the actual presentation of the six stimulus statements and 6 reflecting information processing during the pause interval immediately following each statement. Pupillary reactions during the verbalization period were not included since the study was concerned primarily with the processing stage and since overt responses influence pupillary activity (Simpson \& Paivio, 1968). Each profile was based on 12 within-block raw-score means. These individual profiles were intercorrelated, and the resulting "subjects" matrix was simplified by an obverse principal-components solution. The largest two "subjects" factors were retained for rotation to simple structure after inspection of the distribution of latent roots that revealed a marked drop after the second value. These factors accounted for two-thirds of the estimated common-factor variance. They were considered to reflect two clearly defined strategies for processing information in this personality-impressionformation task. The reasoning is that the "variables" that loaded these factors were actually Ss, and the Ss had been intercorrelated according to their information processing strategies as reflected by their pupillary activity.

Figure 1 illustrates these processing strategies in terms of two mean profiles based on pupillary data from Ss with above-median loadings on the "subject" factors. These two groups of nine and four Ss, respectively, did not differ in sex representation or in terms of resting-level pupillary activity. The Strategy 1 profile is characterized by two clear features, the increment during the response phase following the presentation of the first discrepant item (submissive) and the subsequent decrement of the profile. The increment implies relatively greater processing activity when a change in information regarding the perceived dominance of others is encountered. The decrement in the profile following this peak reflects a reduction of processing activity and suggests that Ss of this type may have given up trying to integrate additional information about the stimulus person after attempting to process the first discrepant item. Such a strategy bears some similarity to the kind of information processing reported to be characteristic of people with "simple conceptual systems" (cf. Schroder, Driver, \& Streufert, 1967).

Several characteristics of the Strategy 2 profile invite comment: The relatively high absolute level in contrast to that of Strategy 1, the marked decrement after the presentation of the third self-referent statement, and the dramatic sustained increment in the profile when discrepant information is encountered. The generally high level can be interpreted in several ways. First, it might mean that Strategy 2 Ss are predisposed to be aroused by performance situations involving an audience. ${ }^{2}$ On the other hand, their high absolute level might be a reflection of their cognitive style. That is, they may have "complex conceptual systems" in the sense that they are prone to deal with information at higher levels of differentiation, discrimination, and integration (cf. Schroder et al, 1967). Although further research is definitely indicated, the latter cognitive-style interpretation appears most plausible in view of the marked decrement in the profile following the third stimulus item. Such a reduction in processing activity would not be expected from audience-sensitive $S$ because, by definition (Paivio, 1965), such people should continue to respond in an aroused manner throughout situations that involve real or potential evaluation. On the other hand, the profile decrement would be expected from complex Ss when the denotations of the first three stimulusitems are carefully considered. These statements represent dominance, orderliness, and nonaffiliation, respectively. Clearly, a complex person, with natural predispositions to process information, should not have to expend much cognitive effort to integrate an item denoting nonaffiliation after first learning that the stimulus person was both dominant and orderly. This interpretation, that the Strategy 2 profile may represent a complex style of processing, is also consistent with the increment in the final part of the profile during the presentation of the three discrepant items of stimulus information. The increment reflécts heightened processing activity, a predictable response for complex Ss under conditions of stimulus complexity.

In sum, careful examination of these processing-strategy profiles, in relation to contiguously associated stimuli, leads to the tentative interpretation that the two information-processing strategies reflect different degrees of conceptual complexity.

As a check on the validity of this interpretation, other response correlates of these strategies were obtained. Two multiple-regression analyses were performed. In each, the criterion, processing strategy, was defined in terms of Ss' loadings on the "subjects" factors. Predictors were syntactic features of the verbalized impressions and trait ratings. The verbalized impression responses were quantified according to the complexity of their integration (Schroder et al, 1967), and also in terms of such syntactic features relevant to personal adjustment as negations and qualifying subordinators (Pylyshyn, 1967). The regression analyses yielded coefficients of .49 and .81 , respectively, neither of which is statistically significant. These results are not surprising since the general problem of identifying overlap between typical pattems of behavior and types of nervous-system activity has been largely refractory (Teplov, 1964). However, the obtained low-power predictions warrant some comment since the set of response measures used as predictors was considerably more inclusive than is customary in studies of impression formation. The major implication of these findings is that inferences about processes involved in personality impression formation, based on traditional manifest behavior variables alone, may not be entirely representative.

Notwithstanding this, the beta weights computed for the predictors in these regression analyses provide some limited support for the former interpretations of the processing strategies. For example, the suggestion that Strategy 1 may be a conceptually simple style of information processing is reinforced by the finding that the highest beta value in the Type 1 regression analysis was associated with the rating scale predictor "strong-weak." The mean rating of 6.3 of Strategy 1 Ss on this variable indicated that they considered the stimulus person to be extremely weak. This suggests a marked recency effect and confirms the above hypothesis that they did not attempt to integrate the conflicting stimulus information.

In a similar way, the interpretation that Strategy 2 may be a complex style of processing received some support from the finding that the highest beta values in the 
second regression analysis were associated with the rating-scale predictors, "secure-insecure" and "reliable-unreliable." The point here is that inferences about the personal security and reliability of the stimulus person involved going beyond the information given, since it may be recalled that the stimulus person was represented solely by information conceming dominance, orderliness, and affiliation. Such a liberal inference style is characteristic of people with complex conceptual systems (Schroder et al, 1967).

\section{REFERENCES}

ASCH, S. E. Forming impressions of personality. Journal of Abnormal \& Social Psychology, $1946,41,258-290$.

HESS, E. H., \& POLT, J. M. Pupil size in relation to mental activity during simple problem solving. Science, 1964, 143, 1190-1192.

JACKSON, D. N. Personality research form manual Goshen, N.Y.: Research Psychologists Press, 1967.

KAHNEMAN, D., \& BEATTY, J. Pupil diameter and load on memory. Science, 1966, 154, 1583-1585.

LAY, C. H., \& JACKSON, D. N. An analysis of the generality of trait inferential networks. Journal of Personality \& Social Psychology, 1969, 12 , 12-21.

PAIVIO, A. Personality and audience influence. In
B. A. Maher (Ed.), Progress in experimental personality research. Vol. 2. New York: Academic Press, 1965.

PAIVIO, A., \& SIMPSON, H. M. The effect of word abstractness and pleasantness on pupil size during an imagery task. Psychonomic Science, 1966, 5, 55-56.

PYLYSHYN, Z. W. Clinical correlates of some syntactic features of patients' speech. Research Bulle tin No. 41, University of Westem Ontario, London, 1967.

SHRODER, H. M., DRIVER, M. J., \& STREUFERT, S. Human information processing. New York: Holt, Rinehart, \& Winston, 1967.

SIMPSON, H. M., \& PAIVIO, A. Effects on pupil size of manual and verbal indicators of cognitive task fulfillment. Perception \& Psychophysics, 1968, 3, 185-190.

TEPLOV, B. M. Problems in the study of general types of higher nervous system activity in man and animals. In J. A. Gray (Ed.), Pavlov's typology. Oxford: Pergamon Press, 1964. Pp. 3-158.

\section{NOTES}

1. This research was partially supported by Canada Council Grant 68-0667 to the first author, and National Research Council Grant AP-304 to the second author, and by the University of Westem Ontario Research Fund.

2. Simpson, H. M. Personal communication. Mean pupillary dilation during a standard cognitive task was significantly larger for a group of high audience-sensitive $S s$ than for low audience-sensitive Ss equated for IQ.

\section{Cooperation and sharing behavior among culturally deprived preschool children}

BARBARA H. WASIK, S. KATHY SENN, and ALEX EPANCHIN, Education Improvement Program, Duke University, Durham, N.C. 27706

Culturally deprived Negro and white kindergarten children $(N=12)$ were paired in like-sex dyads to investigate cooperative and sharing behavior. The development of cooperative behavior was demonstrated in a game situation in which the children received a marble for cooperative responses. Selfish behavior was analyzed using two different definitions which resulted in varying per cents of selfish behavior. A discussion of the disparities ensued.

According to different investigators, preschool children, when given an opportunity to divide objects between themselves and a second person, have demonstrated selfish behavior. That is, they retained more items for themselves than they gave to another child (Ugurel-Semin, 1952; Handlon \& Gross, 1959). To arrange a possible sharing situation, Handlon \& Gross (1959) had dyads of preschool- and elementary-age children play a game in which they were required to cooperate in order to receive a seal. After the children received a specified odd number of seals, each was asked to divide the seals between himself and the other child. The preschool children demonstrated selfish behavior by retaining a mean of $72 \%$ of the seals. The selfish behavior demonstrated by the older children continually declined. Using 4 - to 16-year-old children, Ugurel-Semin (1952) had each child divide an odd number of nuts with another child. On $67 \%$ of the trials, 4 to 6-year-old children showed selfish behavior by keeping more nuts than they gave to the other child. Older children showed less selfish behavior.

In arranging an experiment to study sharing and cooperation behavior among children, it was difficult to interpret research findings such as those above because of differences in definitions and procedures. Although Handlon \& Gross (1959) and Ugurel-Semin (1952) both found preschool children to demonstrate selfish behavior, each gave percentages that represented different experimental definitions of selfish behavior. Handlon \& Gross (1959) defined selfish behavior as the percentage of items kept by the child, while Ugurel-Semin (1952) defined it as the percentage of trials that the $S$ kept more items than he gave to the other child. These two definitions can yield different percentages for the same data and, thus, imply varying results.

Although Handlon \& Gross (1959) required cooperation in a game to lead to the sharing situation, they did not investigate aspects of cooperative behavior. Es such as Azrin \& Lindsley (1956) and Brotsky \& Thomas (1967) have studied cooperative behavior between children in a game situation, but have not studied sharing behavior. Azrin \& Lindsley (1956) demonstrated the development of a cooperative response in children from 7 to 12 years old by requiring two children, who were seated facing each other, to place styli in directly opposite holes within $.04 \mathrm{sec}$ of each other. If successfully completed, a light flashed and a jelly bean dropped into a receptacle between them. Using preschool children, Brotsky \& Thomas (1967) attempted to develop a cooperative response similar to that developed by Azrin \& Lindsley (1956). To cooperate, the children had to press knobs of the same color. A buzz, a light, and an edible treat followed each cooperative response. Although cooperative responses increased, noncooperative responses also increased, and the Es concluded that the children were learning an operant response of "knob pressing" rather than the cooperative response.

The purpose of this study was to arrange a task in which cooperative responses in culturally deprived preschool children could be assessed and sharing of the earned reinforcement could be investigated. Negro and white children were used as $S s$, with like-sex Ss paired in an experimental game. Sharing behavior was analyzed both by the Handlon \& Gross (1959) and the Ugurel-Semin (1952) definitions.

\section{SUBJECTS AND APPARATUS}

The $12 \mathrm{Ss}$ were enrolled in a summer kindergarten for culturally disadvantaged children. Ss, ranging in age from 5.5 to 6.5 years, were randomly selected to fill the squares in a balanced race by sex design. Each $S$ played the game five times, each time with a different partner of the same sex. Within the like-sex groups, all possible combinations of $\mathrm{Ss}$ were used.

The apparatus consisted of two small flat blocks placed on opposite sides of a table. Each block had two button-operated switches, one white switch and either a red or blue switch. On the wall, $2 \mathrm{ft}$ above the experimental table, were the three lights colored red, white, and blue. Seating positions at the side with the red or blue switch were balanced.

Whenever the red switch or the blue switch was pressed, the matching colored light came on for $1 \mathrm{sec}$. When the white switches were pressed simultaneously, the white light came on for $3 \mathrm{sec}$, and a marble rolled into a box in the center of the table. The marbles were dispensed into the room 